\title{
Luteolin and luteolin-7-O-glucoside protect against acute liver injury through regulation of inflammatory mediators and antioxidative enzymes in GalN/LPS-induced hepatitic ICR mice
}

\author{
Chung Mu Park ${ }^{1}$ and Young-Sun Song ${ }^{25}$ \\ 'Department of Clinical Laboratory Science, Dong-Eui University, Busan 47340, Korea \\ ${ }^{2}$ Department of Smart Foods and Drugs, Inje University, 197 Inje-ro, Gimhae, Gyeongnam 50834, Korea
}

BACKGROUND/OBJECTIVES: Anti-inflammatory and antioxidative activities of luteolin and luteolin-7-O-glucoside were compared in galactosamine (GalN)/lipopolysaccharide (LPS)-induced hepatitic ICR mice.

MATERIALS/METHODS: Male ICR mice (6 weeks old) were divided into 4 groups: normal control, GalN/LPS, luteolin, and luteolin-7-O-glucoside groups. The latter two groups were administered luteolin or luteolin-7-O-glucoside (50 mg/kg BW) daily by gavage for 3 weeks after which hepatitis was induced by intraperitoneal injection of GalN and LPS (1 g/kg BW and 10 $\mu \mathrm{g} / \mathrm{kg}$ BW, respectively).

RESULTS: GaIN/LPS produced acute hepatic injury by a sharp increase in serum AST, ALT, and TNF-a levels, increases that were ameliorated in the experimental groups. In addition, markedly increased expressions of cyclooxygenase (COX)- 2 and its transcription factors, nuclear factor (NF)- $\kappa B$ and activator protein (AP)-1, were also significantly attenuated in the experimental groups. Compared to luteolin-7-O-glucoside, luteolin more potently ameliorated the levels of inflammatory mediators. Phase II enzymes levels and NF-E2 p45-related factor (Nrf)-2 activation that were decreased by GalN/LPS were increased by luteolin and luteolin-7-O-glucoside administration. In addition, compared to luteolin, luteolin-7-O-glucoside acted as a more potent inducer of changes in phase II enzymes. Liver histopathology results were consistent with the mediator and enzyme results. CONCLUSION: Luteolin and luteolin-7-O-glucoside protect against GaIN/LPS-induced hepatotoxicity through the regulation of inflammatory mediators and phase II enzymes.

Nutrition Research and Practice 2019;13(6):473-479; https://doi.org/10.4162/nrp.2019.13.6.473; pISSN 1976-1457 elSSN 2005-6168

Keywords: Luteolin, Luteolin-7-O-glucoside, Inflammation, NF-kappa B, NF-E2-Related Factor 2

\section{INTRODUCTION}

Luteolin (3', 4',5,7-hydroxyl-flavone) is a naturally occurring flavone in plants. Interest in the possible health benefits of flavones has increased due to their reported potent antiinflammatory and antioxidative activities in vitro and in vivo $[1,2]$. Flavones in plants are usually present in the form of glucosides (sugar attached), although occasionally they are found as aglycones. It is known that glucosides are hydrolyzed by the $\beta$-glucosidase enzyme produced by gut bacteria and are subsequently absorbed as aglycones [3]. Aglycones are absorbed faster and in greater quantities than their glucoside counterparts in vivo [4,5], whereas glucosides can be partially absorbed (and remain biologically active) without $\beta$-glucosidase hydrolysis [6].

After absorption, aglycones mostly undergo intestinal conjugation to mono-glucuronide or hepatic sulfation/methylation and, thus, are metabolized as various forms of glucuronides, sulfates, or methylates [7] or remain as unmetabolized aglycones and glucosides at low levels [8]. There are several pieces of evidence that indicate the bioavailability of flavones may differ when consumed as either aglycones or glucosides [4,9], and there is also contradictory evidence that the bioavailability of flavones does not differ when consumed as either aglycones or glycosides $[10,11]$. However, recent research has shown that the type of metabolites transformed after absorption may determine the bioactivity of flavones [8]. That is, depending on the bioavailability of flavones and the transformation pattern to certain metabolites, biological activities of aglycone and glucoside forms of flavones could be different in vivo.

Galactosamine/lipopolysaccharide (GalN/LPS) is a well-known model for acute liver injury that closely resembles human clinical hepatitis [12]. GalN is a hexosamine derived from galactose and has a molecular formula of $\mathrm{C}_{6} \mathrm{H}_{13} \mathrm{NO}_{5}$. This amino sugar is hepa-

\footnotetext{
This research was supported by the Basic Science Research Program through the National Research Foundation of Korea (NRF), funded by the Ministry of Education, Science, and Technology (NRF-2017R1D1A3B03031997).

${ }^{\S}$ Corresponding Author: Young-Sun Song, Tel. 82-55-320-3235; Fax. 82-55-321-0691, Email. fdsnsong@inje.ac.kr

Received: April 23, 2019, Revised: May 24, 2019, Accepted: July 15, 2019

This is an Open Access article distributed under the terms of the Creative Commons Attribution Non-Commercial License (http://creativecommons.org/licenses/by-nc/3.0/) which permits unrestricted non-commercial use, distribution, and reproduction in any medium, provided the original work is properly cited.
} 
totoxic; the toxic effect of GalN is connected with insufficiency of UDP-glucose and UDP-galactose and the loss of intracellular calcium homeostasis. These changes affect cell membranes and organelles, as well as the synthesis of proteins and nucleic acids [13]. LPS is a major component of the outer membrane of Gram-negative bacteria and triggers excessive secretion of various inflammatory mediators such as nitric oxide (NO) and prostaglandin $E_{2}\left(P G E_{2}\right)$, which are regulated by transcription factors such as nuclear factor- $\kappa \mathrm{B}$ (NF- $\kappa \mathrm{B})$ and activator protein-1 (AP-1) [14]. Such factors reside ubiquitously in the cytoplasm as heterodimers with p50 and p65 as well as with c-jun and fos. In response to an inflammatory stimulus, transcription factors are activated and translocated into the nucleus resulting in the upregulation of inducible NO synthase (iNOS) and cyclooxygenase-2 (COX-2), which are the corresponding enzymes of $\mathrm{NO}$ and $\mathrm{PGE}_{2}[14,15]$. Moreover, LPS generates reactive oxygen species and thus increases oxidative stress. The buildup of oxidative stress is ameliorated through the modulation of an antioxidative transcription factor, nuclear factor-erythroid 2 p45-related factor-2 (Nrf-2), mediating phase 2 enzyme expressions [16]. Nrf2 is known as a major determinant for the induction of phase 2 enzymes, such as heme oxygenase-1 (HO-1), $\mathrm{NAD}(\mathrm{P}) \mathrm{H}$ :quinone oxidoreductase 1 (NQO1), and superoxide dismutase (SOD). This transcription factor is also ubiquitously present in cytoplasm that is anchored by the negative regulator Kelch-like ECH associated protein 1 (Keap1). Under inflammatory circumstances, Nrf2 dissociates from Keap1 and accumulates in the nucleus, binding with antioxidant response elements in the promoter region of various phase 2 enzymes. [17]. Therefore, GalN/LPS challenge is a good approach to inducing inflammation and oxidative stress in a hepatic failure animal model and is useful in the study of the anti-inflammatory and antioxidative activities of phytochemicals in vivo [18].

Numerous previous studies have shown that luteolin aglycone is an anti-inflammatory and antioxidative agent in vitro and in vivo, whereas the bioavailability of luteolin and its glycosidic form has not not yet been fully described. [1,19-23]. This study was designed to compare the anti-inflammatory and antioxidative activities of luteolin and luteolin-7-O-glucoside (a major glucoside present in plants) as well as their underlying molecular mechanisms in GalN/LPS-induced hepatotoxic ICR mice.

\section{MATERIALS AND METHODS}

\section{Reagents}

Luteolin, GaIN, LPS, and SDS were purchased from the Sigma Chemical Co. (St. Louis, MO, USA). Luteolin-7-O-glucoside was obtained from the INDOFINE chemical company (Hillsborough, NJ, USA). Antibodies against COX-2, phospho-p65, phospho-c-jun, HO-1, NQO1, SOD-1, SOD-2, catalase, Nrf-2, and actin, as well as horseradish peroxidase (HRP)-conjugated anti-rabbit IgG, were purchased from Cell Signaling Technology (Danvers, MA, USA) and Santa Cruz Biotechnology (Santa Cruz, CA, USA). Polyvinylidene difluoride (PVDF) membrane was supplied by Bio-Rad Laboratories (Hercules, CA, USA). All other chemicals were of the highest commercial grade available.

\section{Animals}

Six-week-old male ICR mice were purchased from an experimental animal breeder, Samtako Bio Korea (Osan, Korea), and acclimated to laboratory conditions for one week. The animals were housed individually in suspended plastic cages and maintained in conditions of $22-25^{\circ} \mathrm{C}, 50-60 \%$ relative humidity, and a $12 \mathrm{~h}$ light/dark cycle with free access to food (AIN-93G diet) and water. In order to compare the protective effects of luteolin and luteolin-7-O-glucoside against LPS/GalN-induced acute liver injury, the animals were divided into 4 groups of 8 mice each: normal control group, GalN/LPS group, luteolin, and luteolin-7-O-glucoside groups. The animals in the latter two groups were orally administered with luteolin or luteolin-7O-glucoside (50 mg/kg BW dissolved in 0.5\% CMC-Na solution) daily for 3 weeks before hepatitis induction, while the normal control and GalN/LPS groups received equal volumes of distilled water or $0.5 \%$ CMC-Na solution, respectively, by gavage for the same duration. On the last day, GalN and LPS ( $1 \mathrm{~g} / \mathrm{kg}$ BW and $10 \mu \mathrm{g} / \mathrm{kg}$ BW, respectively) were intraperitoneally administered to all groups except the normal control group [24]. After 24 $h$, the ICR mice were anesthetized by carbon dioxide and sacrificed. Their blood and livers were collected and preserved at $-70^{\circ} \mathrm{C}$ until biological assays were performed. All animal experiments were approved by the Committee of Laboratory Animals in accordance with the Institutional Guidelines of Dong-Eui University, Republic of Korea (IACUC-R2017-013).

\section{Hepatic enzymes activities}

Mouse blood samples were held at room temperature for 1 $h$, after which serum was obtained by centrifugation at 1,000 $\times \mathrm{g}$ for $10 \mathrm{~min}$. Activity levels of aspartate aminotransferase (AST) and alanine aminotransferase (ALT) were measured by using a Mindray BS-120 chemical analyzer (Shenzen Mindray Bio-Medical Electronics, Shenzen, China).

\section{Serum TNF- $a$ concentration}

A mouse tumor necrosis factor (TNF)-a ELISA kit was employed to determine the serum TNF- $a$ concentration according to the manufacturer's instructions (R\&D Systems, Minneapolis, MN, USA).

\section{Western blot analysis}

Protein from liver tissue was obtained by using a protein extraction solution (Intron Biotechnology, Seongnam, Korea). Briefly, $20 \mathrm{mg}$ of fresh liver tissue was homogenized into 0.4 $\mathrm{mL}$ of ice-cold protein extraction solution and lysed for $10 \mathrm{~min}$ at $-20^{\circ} \mathrm{C}$. After $10 \mathrm{~min}$, the disrupted tissue was centrifuged at $13,000 \times \mathrm{g}$ for $10 \mathrm{~min}$ at $-4^{\circ} \mathrm{C}$. Protein concentration was determined by performing a Bradford assay. One hundred micrograms of protein sample were separated on $10 \%$ SDSpolyacrylamide gel and electrotransferred to a PVDF membrane (Bio-Rad Laboratories). Membranes were blocked for $1 \mathrm{~h}$ at room temperature with $5 \%$ nonfat dry milk in a TBST solution. The reactions were then incubated at $4^{\circ} \mathrm{C}$ overnight with 1:1,000 dilution of individual primary antibodies. After overnight incubation, the membranes were washed and then incubated with a 1:1,000 dilution of HRP-conjugated anti-rabbit IgG for $2 \mathrm{~h}$ at room temperature. Blots were developed by using an 
ECL developing solution (Santa Cruz Biotechnology), and the data were quantified using the Gel Doc EQ System (Bio-Rad Laboratories). All signals were normalized to protein levels of the housekeeping gene actin and are expressed as ratios.

\section{Histopathological observation}

The livers were immediately fixed in $10 \%$ formalin and embedded in paraffin wax. Sections were cut at a $5 \mu \mathrm{m}$ thickness and stained with hematoxylin and eosin. Histopathological changes were examined under a light microscope.

\section{Statistical analysis}

All data are expressed as mean \pm standard deviation (SD) values. The statistical analyses were performed using SPSS version 10.0 (SPSS Institute, Chicago, IL, USA). One-way ANOVA with Duncan's multiple-range test was used to examine the differences between groups. $P$ values of $<0.05$ were considered significant, if not stated otherwise.

\section{RESULTS}

Luteolin and luteolin-7-O-glucoside ameliorated serum AST and $A L T$ activities in GalN/LPS-intoxicated mice.

Serum AST and ALT activities were analyzed in order to identify and compare the protective effects of luteolin and luteolin-7-O-glucoside against GalN/LPS-induced hepatic injury. As shown in Fig. 1, both hepatic enzymes activity levels were sharply increased as a result of GalN/LPS exposure, and those increases were significantly ameliorated in the luteolin and luteolin-7-O-glucoside administrated groups. In a comparison of the two flavones, luteolin exhibited more potent protective activity than that of luteolin-7-O-glucoside in GalN/LPS-intoxicated ICR mice.

Luteolin and luteolin-7-O-glucoside reduced inflammatory features in GalN/LPS-induced hepatitis mice.

Histopathological changes in the livers of each of the four groups can be seen in Fig. 2. GalN/LPS injection produced

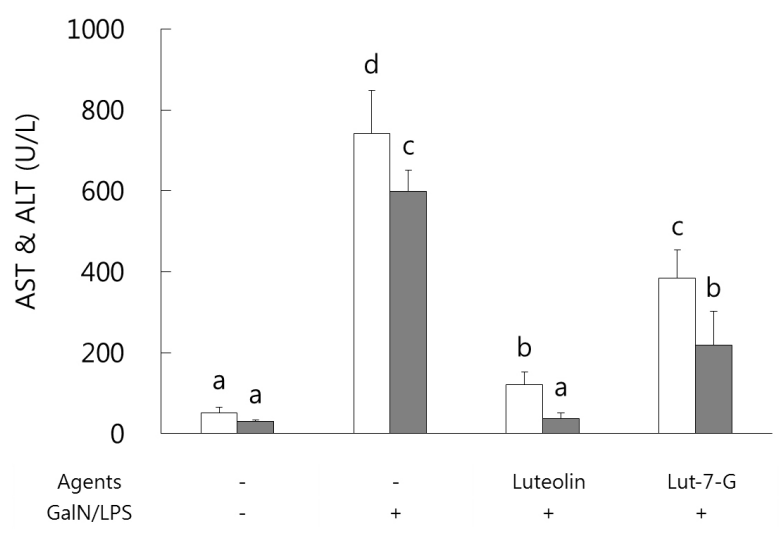

Fig. 1. Effect of luteolin and luteolin 7-O-glucoside on serum AST and ALT activity levels in GalN/LPS-intoxicated ICR mice. AST and ALT activities were attenuated in luteolin and luteolin-7-O-glucoside administered groups of GalN/LPS intoxicated ICR mice. Values sharing the same superscript are not significantly different at $P<0.05$ (Duncan's multiple-range test), Lut-7-G, luteolin-7-O-glucoside; GalN galactosamine; AST, aspartate aminotransferase; ALT, alanine aminotransferase.
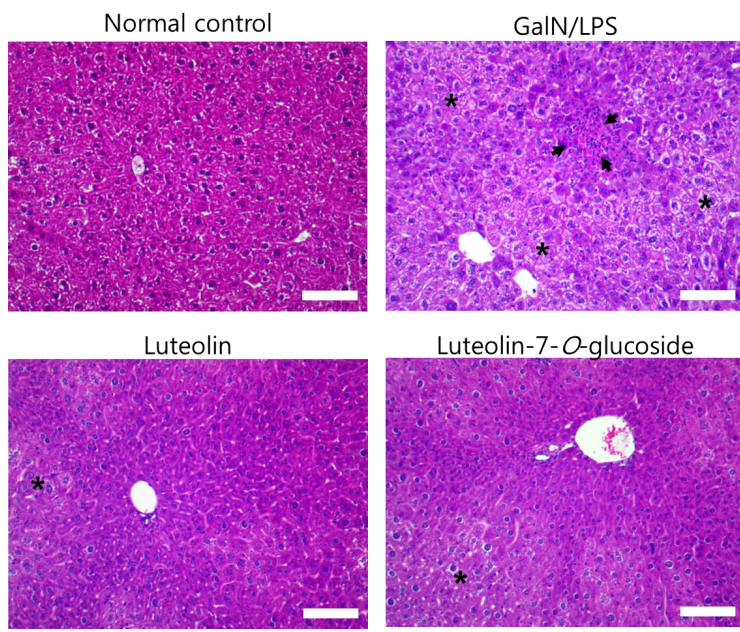

Fig. 2. Effect of luteolin and luteolin 7-O-glucoside on histopathological changes in liver of mice treated with GaIN/LPS. Liver tissue sections were stained with hematoxylin and eosin. The arrows indicate inflammatory cell infiltration and the asterisks exhibit necrosis in the liver tissue of GalN/LPS-intoxicated mouse. Magnification, 200×; scale bar, $100 \mu \mathrm{m}$.
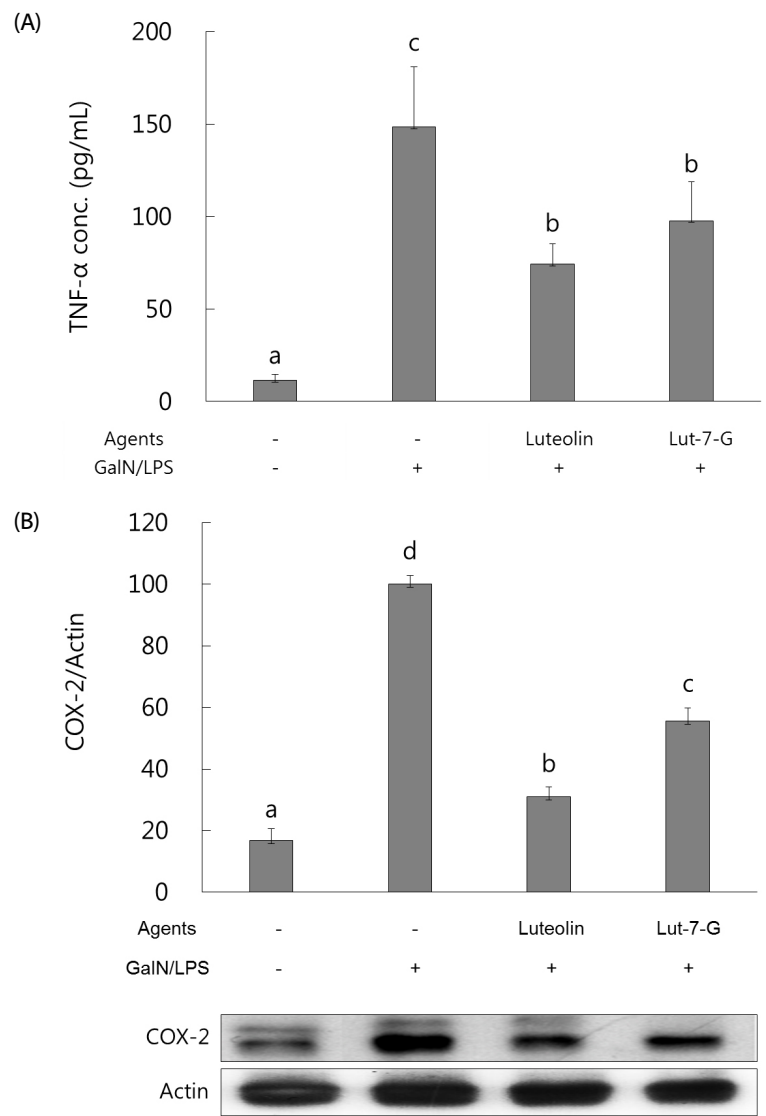

Fig. 3. Effect of luteolin and luteolin 7-O-glucoside on serum TNF-a concentration and COX-2 expression in GaIN/LPS-intoxicated ICR mice. Serum TNF-a concentration (A) and COX-2 protein expression (B) were analyzed by ELISA assay and western blot analysis, respectively. The data are representative of three independent experiments. The relative induction of COX-2 protein expression was quantified by densitometry with actin used as an internal control. The data represent the mean \pm standard deviation of triplicate experiments. Values sharing the same superscript are not significantly different at $P<0.05$ (Duncan's multiple-range test). Lut-7-G, Iuteolin-7-O-glucoside; TNF, tumor necrosis factor: GalN, galactosamine; LPS, lipopolysaccharide; COX, cyclooxygenase. 


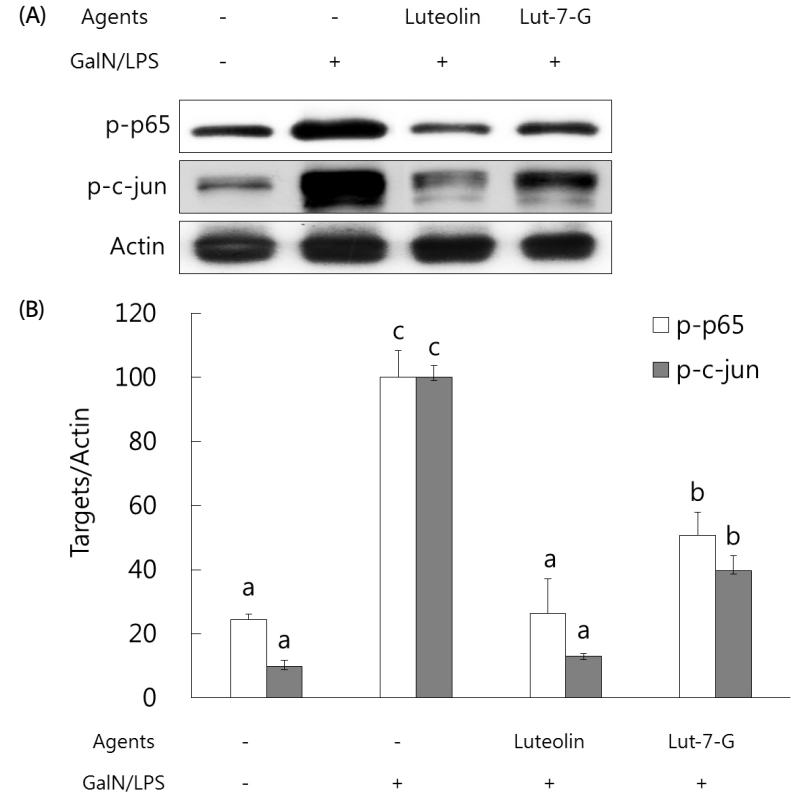

Fig. 4. Effect of luteolin and luteolin 7-O-glucoside on the phosphorylation status of the inflammatory transcription factors NFkB and AP-1 in GalN/LPS-intoxicated ICR mice. (A) The phosphorylated status of p65 and c-jun, subunits of NFKB and AP-1, were analyzed by western blot analysis. The data are representative of three independent experiments. (B) The relative induction of p65 and c-jun phosphorylation was quantified by densitometry with actin used as an internal control. The data represent the mean \pm standard deviation of triplicate experiments. Values sharing the same superscript are not significantly different at $P<0.05$ (Duncan's multiple-range test). Lut-7-G, luteolin-7-O glucoside; GalN, galactosamine; LPS, lipopolysaccharide.

inflammatory cell infiltration and necrosis compared to that in the normal control group. However, these changes were attenuated by the 3-week administration of luteolin or luteolin7-O-glucoside.

Luteolin and luteolin-7-O-glucoside attenuated inflammatory mediators and their transcription factors, NF- $\kappa B$ and $A P-1$, in GalN/LPS-intoxicated mice.

$\mathrm{NF}-\mathrm{KB}$ and $\mathrm{AP}-1$ have a critical role in the regulation of inflammatory mediators such as TNF- $a$ and COX-2. In order to measure their levels, ELISA assay and western blot analysis were employed. As shown in Fig. 3, serum TNF-a concentration and hepatic COX-2 expression were markedly elevated with GalN/ LPS exposure. However, luteolin and luteolin-7-O-glucoside administration significantly ameliorated the increased TNF- $a$ production and COX-2 expression. To identify whether these alterations might occur through NF- $\kappa \mathrm{B}$ and AP-1 regulation, the phosphorylated status of both transcription factors were measured by western blot analysis. As shown in Fig. 4, the GalN/LPS-induced activations of p65 and c-jun, subunits of NF- $\kappa$ $B$ and AP-1, respectively, were attenuated by luteolin and luteolin-7-O-glucoside administration. The levels of the inflammatory mediators and their transcription factors were more potently ameliorated in the luteolin-treated group than in the luteolin-7-O-glucoside-administered group.

Luteolin and luteolin-7-O-glucoside restored activities of phase II enzymes and their transcription factor, Nrf-2, in GalN/LPS-

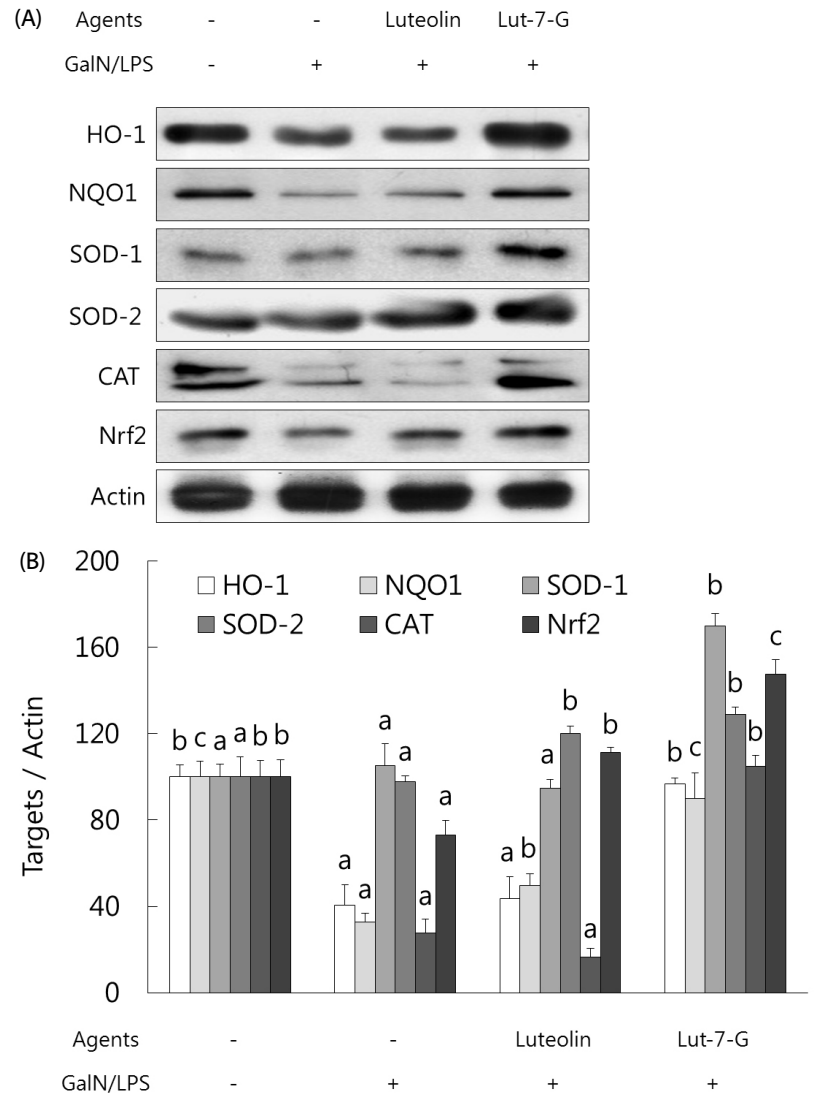

Fig. 5. Effect of luteolin and luteolin 7-O-glucoside on phase II enzymes and their transcription factor, Nrf2, in GalN/LPS-intoxicated ICR mice. (A) The phase II enzymes and Nrf2 expressions were analyzed by western blot analysis. The data are representative of three independent experiments. (B) The relative induction of protein expression was quantified by densitometry with actin used as an internal control. The data represent the mean \pm standard deviation of triplicate experiments. Values sharing the same superscript are not significantly different at $P<0.05$ (Duncan's multiple-range test). Lut-7-G, Iuteolin-7-O-glucoside; Nrf2, nuclear factor E2-related factor 2; GalN, galactosamine; LPS, lipopolysaccharide; HO-1, heme oxygenase-1; NQO, quinone oxidoreductase; SOD, superoxide dismutase; CAT, catalase.

\section{intoxicated mice.}

Expression of phase II enzymes was measured by performing western blot analysis due to their anti-inflammatory activity. As shown in Fig. 5, GalN/LPS administration produced a sharp reduction in the activities of phase II enzymes activities such as HO-1, NQO1, SOD-1, SOD-2, and catalase in accordance with the inhibition of their transcription factor, Nrf-2. Administration of luteolin or luteolin-7-O-glucoside for 3 weeks significantly enhanced the phase II enzymes and Nrf-2 activities. Compared to luteolin, luteolin-7-O-glucoside more potently induced phase II enzymes activities in GalN/LPS-intoxicated mice. These results indicate that both luteolin and luteolin-7-O-glucoside protect against acute liver injury through attenuation of inflammatory mediators and enhancement of phase II enzymes in GaIN/LPSinduced hepatitic mice.

\section{DISCUSSION}

The anti-inflammatory and antioxidative activities of luteolin and luteolin-7-O-glucoside were compared in GaIN/LPS-induced 
hepatotoxic mice. Hepatic injury upon GaIN/LPS exposure was confirmed by the elevated AST and ALT levels (Fig. 1) as well as the presence of infiltrated inflammatory cells (Fig. 2). Moreover, the concentration of plasma TNF- $a$ and the level of hepatic COX-2 expression, major inflammatory mediators by GalN/LPS, were also elevated in the GalN/LPS group. These data support the suggestion that GalN/LPS exposure to mice is a good method to develop an acute liver injury. Experimental models of oxidative stress and inflammation-mediated acute liver injury have been induced via the administration of LPS [23], GalN [25], and acetaminophen [26]. LPS triggers an excessive secretion of inflammatory mediators through the regulation of inflammatory transcription factors, TLR-mediated NF- $\kappa B$ and AP-1 [18,21,27]. In addition, GalN exerts hepatotoxicity by inhibiting the biosynthesis of RNA and proteins [25]. In an attempt to cope with the relative insensitivity of rodents to LPS, GalN markedly sensitizes mice to LPS [28], and thus, GalNsensitized mice exhibit increased sensitivities to the toxic effects of TLR ligands by LPS [29]. On that basis, GalN/LPS challenge seems to an effective model of hepatotoxicity that closely resembles acute liver failure by successfully triggering inflammation and oxidative stress. However, there is a report that there is a difference between LPS-induced systemic inflammation and the hepatic injury induced by GaIN/LPS administration [30]. They observed that TNF- $a$ was a key mediator in the GalN/LPS system, causing hepatic apoptosis and subsequent necrosis [4], which were also observed in this study (Fig. 1 and Fig. 2).

The administration of luteolin or luteolin-7-O-glucoside protected against hepatic injury by GaIN/LPS as was evidenced by the lowered AST and AST activity levels. Liver microstructural observations of a reduced incidence of inflammatory cell infiltration and the presence of apoptosis and necrosis supported the presence of hepatoprotective effects of luteolin and luteolin7-O-glucoside in GalN/LPS-injected mice. Anti-inflammatory effects of luteolin and luteolin-7-O-glucoside were confirmed by the amelioration of the levels of serum TNF-a concentration and hepatic COX-2 expression. Moreover, activations of p65 and c-jun by GalN/LPS, subunits of NF- $k B$ and AP-1, were attenuated by both luteolin and luteolin-7-O-glucoside administration. Activation of NF- $\kappa B$ and AP-1 is highly associated with the induction of inflammatory enzymes, including COX-2 [14,31]. Thus, suppression of TNF- $a$ and COX-2 levels might be mediated through the inhibition of NF- $\kappa B$ and AP-1 transactivation by both luteolin and luteolin-7-O-glucoside. These mechanisms of anti-inflammatory activities of luteolin have been studied in vitro [21]. Luteolin protects against acute lung injury by LPS through the inhibition of the MEK/ERK and PI3K/Akt pathways in neutrophils [32] and inhibition of iNOS expression by inactivating NF- $\kappa B$ and AP-1 by TNF-a via the JNK pathway in HepG2 cells [33]. These findings indicate that the upstream signaling pathways that modulate transcription factors might differ depending on the inducer or cell line used. Our previous study in RAW 264.7 cells showed that luteolin attenuated the activation of both NF- $\mathrm{BB}$ and AP-1, while luteolin-7-O-glucoside impeded only NF- $\kappa B$ activation, which might be attributed to differential activation of the NF- $\mathrm{kB} / \mathrm{AP}-1 / \mathrm{PI} 3 \mathrm{~K}$-Akt pathway in vitro [34]. However, this in vivo study showed that both luteolin and luteolin-7-O-glucoside inhibited the activation of NF- $\kappa \mathrm{B}$ and
AP-1. The discrepancy between the in vitro and in vivo mechanisms may be related to the metabolic fates of luteolin and luteolin-7-O-glucoside. Luteolin glucoside is hydrolyzed to free luteolin in the gastrointestinal tract by microorganisms and then converted to luteolin glucuronides or other metabolites [35]. Unmetabolized luteolin and luteolin-7-O-glucoside are present at a very low level [8], and the predominant metabolite in blood and tissues is luteolin-3'-O-glucuronide, which was shown to have an anti-inflammatory effect in RAW 264.7 cells [8]. Therefore, the mechanism involved in the anti-inflammatory effects of luteolin and luteolin-7-O-glucoside in vitro or by luteolin-3'-O-glucuronide in vivo seems to be somewhat different.

In the comparison of the anti-inflammatory effects of luteolin and luteolin-7-O-glucoside, luteolin exhibited a more potent anti-inflammatory activity than luteolin-7-O-glucoside in GalN/ LPS-intoxicated ICR mice. Suppression of NF-kB and AP-1 by luteolin was stronger than that induced by luteolin-7-O-glucoside. These findings might partly explain why luteolin-7-O-glucoside weakly mitigated LPS-induced TNF-a concentration and COX-2 expression when compared to the strong suppression obtained with luteolin administration. This might be due to variation in the cellular uptakes of luteolin and luteolin-7-O-glucoside [22]. Murota et al. [35] found that the aglycone form of flavone was taken up into Caco-2 cells more efficiently than the glycoside form because of its more moderate lipophilicity. Several researchers also have confirmed that flavonoid glycosides are not easily absorbed because they are bound to sugars such as $\beta$-glucosides $[4,8,34,35]$. Contrarily, there are suggestions that flavonoid glycosides have shown similar or greater uptake efficacy than that of their corresponding aglycones in human studies $[11,36,37]$. However, a recent study on the metabolic fate of luteolin and luteolin-7-O-glucoside in rats confirmed that the bioavailability of luteolin is higher than that of luteolin-7O-glucoside in rats [8]. They also found that luteolin is absorbed faster than luteolin-7-O-glucoside and the area under the curve of plasma luteolin-3-O-glucuronide (their major metabolite) for $24 \mathrm{~h}$ after oral administration was bigger in the luteolin group than in the luteolin-7-O-glucoside group, implying a higher absorption rate for luteolin than that for luteolin-7-O-glucoside. These results support our data and suggest why luteolin more potently ameliorated the levels of inflammatory mediators and transcription factors than that by luteolin-7-O-glucoside.

Antioxidative effects of luteolin and luteolin-7-O-glucoside were confirmed by the upregulation of phase II enzymes expressions (Fig. 5). GalN/LPS administration produced a sharp reduction in phase II enzymes expressions, such as $\mathrm{HO}-1, \mathrm{NQO1}$ SOD-1, SOD-2, and catalase, in accord with inhibition of their transcription factor, Nrf2. Nrf-2 is a transcription factor and induces expression of antioxidant response element-dependent genes such as HO-1, NQO1, SOD-1, SOD-2, and catalase [16]. Administration of luteolin-7-O-glucoside significantly enhanced the phase II enzymes and Nrf-2 expressions, whereas luteolin enhanced only SOD-2 and Nrf-2 expressions. Thus, both luteolin and luteolin-7-O-glucoside ameliorate oxidative stress from GalN/LPS treatment through modification of phase II enzymes and their transcription factor, Nrf-2. Comparison of the two flavones showed that luteolin-7-O-glucoside more potently induced phase II enzymes expressions than those of luteolin 
in GalN/LPS-intoxicated mice, which means that luteolin-7-Oglucoside has stronger antioxidative activity than that of luteolin. Our previous in vitro study showed that luteolin had greater antioxidant potential than luteolin-7-O-glucoside via the modulation of the Nrf-2 mediated $\mathrm{HO}-1$ signaling cascade in RAW 264.7 cells [22]. In a comparison of the reactive oxygen species scavenging activities of luteolin and luteolin-7-Oglucoside against t-butyl hydroperoxide-induced oxidative damage, both luteolin and luteolin-7-O-glucoside had strong antioxidant potential [22].

In this study, we assayed phase II enzymes and Nrf-2 expressions in the liver. The data from Kure et al. [8] may help explain our results. They reported that the predominant metabolite in blood and tissues, including kidney and small intestine, is luteolin-3'-O-glucuronide and its concentration was higher in their luteolin group than in their luteolin-7-O-glucoside group. However, hepatic luteolin-3'-O-glucuronide and total glucuronide concentrations, including luteolin-3'-O-glucuronide, luteolin-4'O-glucuronide and luteolin-7'-O-glucuronide, was higher in their luteolin-7-O-glucoside group $(0.55 \mu \mathrm{g} / \mathrm{g}$ and $1.30 \mu \mathrm{g} / \mathrm{g}$, respectively, in liver) than in their luteolin group $(0.37 \mu \mathrm{g} / \mathrm{g}$ and 0.87 $\mu \mathrm{g} / \mathrm{g}$, respectively, in liver) [8]. It is unclear what type of luteolin-glucuronide has a strong antioxidant potential against oxidative stress. Nonetheless, luteolin glucuronides, not luteolin or luteolin-7-O-glucoside, are presumed to be responsible for antioxidative activity in vivo.

Taken together, our results strongly suggest that luteolin and luteolin-7-O-glucoside protect against acute liver injury through the regulation of inflammatory mediators and phase II enzymes in GalN/LPS-induced hepatotoxic mice. However, luteolin and luteolin-7-O-glucoside had slightly different efficacy against inflammation and oxidative stress. Our results suggest that either luteolin aglycone or its glucosides might be potential candidate therapeutics in the treatment of inflammation and oxidative stress. Further research to compare the efficacy of luteolin and its glucosides in various animal models is required to fully elucidate the bioavailability and metabolism of flavones.

\section{CONFLICT OF INTEREST}

The authors declare no potential conflicts of interest.

\section{ORCID}

Chung Mu Park: https://orcid.org/0000-0001-8477-6276

Young-Sun Song: https://orcid.org/0000-0002-8190-4815

\section{REFERENCE}

1. Fei J, Liang $B$, Jiang $\mathrm{C}$, Ni H, Wang L. Luteolin inhibits IL-1 $\beta$-induced inflammation in rat chondrocytes and attenuates osteoarthritis progression in a rat model. Biomed Pharmacother 2019;109: 1586-92.

2. Seelinger G, Merfort I, Schempp CM. Anti-oxidant, anti-inflammatory and anti-allergic activities of luteolin. Planta Med 2008;74:1667-77.

3. Richelle M, Pridmore-Merten S, Bodenstab S, Enslen M, Offord EA. Hydrolysis of isoflavone glycosides to aglycones by beta-glycosidase does not alter plasma and urine isoflavone pharmacokinetics in postmenopausal women. J Nutr 2002;132:2587-92.

4. Izumi T, Piskula MK, Osawa S, Obata A, Tobe K, Saito M, Kataoka S, Kubota Y, Kikuchi M. Soy isoflavone aglycones are absorbed faster and in higher amounts than their glucosides in humans. J Nutr 2000;130:1695-9.

5. Kano M, Takayanagi T, Harada K, Sawada S, Ishikawa F. Bioavailability of isoflavones after ingestion of soy beverages in healthy adults. J Nutr 2006;136:2291-6.

6. Andlauer W, Kolb J, Fürst P. Isoflavones from tofu are absorbed and metabolized in the isolated rat small intestine. J Nutr 2000;130: 3021-7.

7. Rowland I, Faughnan M, Hoey L, Wähälä K, Williamson G, Cassidy A. Bioavailability of phyto-oestrogens. Br J Nutr 2003;89 Suppl 1:S45-58.

8. Kure A, Nakagawa K, Kondo M, Kato S, Kimura F, Watanabe A, Shoji $\mathrm{N}$, Hatanaka S, Tsushida T, Miyazawa T. Metabolic fate of luteolin in rats: its relationship to anti-inflammatory effect. J Agric Food Chem 2016;64:4246-54.

9. Zhou P, Li LP, Luo SQ, Jiang HD, Zeng S. Intestinal absorption of luteolin from peanut hull extract is more efficient than that from individual pure luteolin. J Agric Food Chem 2008;56:296-300.

10. Piskula MK. Factors affecting flavonoids absorption. Biofactors 2000;12:175-80

11. Zubik L, Meydani M. Bioavailability of soybean isoflavones from aglycone and glucoside forms in American women. Am J Clin Nutr 2003;77:1459-65.

12. Kemelo MK, Wojnarová L, Kutinová Canová N, Farghali H. D-galactosamine/lipopolysaccharide-induced hepatotoxicity downregulates sirtuin 1 in rat liver: role of sirtuin 1 modulation in hepatoprotection. Physiol Res 2014;63:615-23.

13. Ferencíková R, Cervinková Z, Drahota Z. Hepatotoxic effect of D-galactosamine and protective role of lipid emulsion. Physiol Res 2003;52:73-8.

14. Surh YJ, Chun KS, Cha HH, Han SS, Keum YS, Park KK, Lee SS. Molecular mechanisms underlying chemopreventive activities of anti-inflammatory phytochemicals: down-regulation of COX-2 and iNOS through suppression of NF-kappa B activation. Mutat Res 2001;480-481:243-68.

15. Fujioka S, Niu J, Schmidt C, Sclabas GM, Peng B, Uwagawa T, Li Z, Evans DB, Abbruzzese JL, Chiao PJ. NF-kappaB and AP-1 connection: mechanism of NF-kappaB-dependent regulation of AP-1 activity. Mol Cell Biol 2004;24:7806-19.

16. Sajadimajd S, Khazaei M. Oxidative stress and cancer: the role of Nrf2. Curr Cancer Drug Targets 2018;18:538-57.

17. Zhang M, An C, Gao Y, Leak RK, Chen J, Zhang F. Emerging roles of Nrf2 and phase II antioxidant enzymes in neuroprotection. Prog Neurobiol 2013;100:30-47.

18. Mao J, Yi M, Wang R, Huang Y, Chen M. Protective effects of costunolide against D-galactosamine and lipopolysaccharide-induced acute liver injury in mice. Front Pharmacol 2018;9:1469.

19. Aziz N, Kim MY, Cho JY. Anti-inflammatory effects of luteolin: a review of in vitro, in vivo, and in silico studies. J Ethnopharmacol 2018;225:342-58

20. Jia Z, Nallasamy P, Liu D, Shah H, Li JZ, Chitrakar R, Si H, McCormick J, Zhu H, Zhen W, Li Y. Luteolin protects against vascular inflammation in mice and TNF-alpha-induced monocyte adhesion to endothelial cells via suppressing IKBa/NF- $\mathrm{kB}$ signaling pathway. J Nutr Biochem 2015;26:293-302. 
21. Park CM, Song YS. Luteolin and luteolin-7-O-glucoside inhibit lipopolysaccharide-induced inflammatory responses through modulation of NF-kB/AP-1/PI3K-Akt signaling cascades in RAW 264.7 cells. Nutr Res Pract 2013;7:423-9.

22. Song YS, Park CM. Luteolin and luteolin-7-O-glucoside strengthen antioxidative potential through the modulation of Nrf2/MAPK mediated HO-1 signaling cascade in RAW 264.7 cells. Food Chem Toxicol 2014;65:70-5.

23. Lee WC, Jung HA, Choi JS, Kim YS, Lee SM. Protective effects of luteolin against apoptotic liver damage induced by D-galactosamine/ lipopolysaccharide in mice. J Nat Prod 2011;74:1916-21.

24. Hossen MJ, Yang WS, Kim D, Aravinthan A, Kim JH, Cho JY. Thymoquinone: an IRAK1 inhibitor with in vivo and in vitro anti-inflammatory activities. Sci Rep 2017;7:42995.

25. Wu YH, Hu SQ, Liu J, Cao HC, Xu W, Li YJ, Li ப. Nature and mechanisms of hepatocyte apoptosis induced by D-galactosamine/ lipopolysaccharide challenge in mice. Int J Mol Med 2014;33: 1498-506.

26. Shanmugam $S$, Thangaraj $P$, Lima $B D$, Chandran $R$, de Souza Araújo AA, Narain N, Serafini MR, Júnior $\amalg$. Effects of luteolin and quercetin 3- $\beta$-d-glucoside identified from Passiflora subpeltata leaves against acetaminophen induced hepatotoxicity in rats. Biomed Pharmacother 2016;83:1278-85.

27. Jiang $W$, Sun $R$, Wei $H$, Tian Z. Toll-like receptor 3 ligand attenuates LPS-induced liver injury by down-regulation of toll-like receptor 4 expression on macrophages. Proc Natl Acad Sci U S A 2005;102: 17077-82.

28. Nowak M, Gaines GC, Rosenberg J, Minter R, Bahjat FR, Rectenwald J, MacKay SL, Edwards CK 3rd, Moldawer LL. LPS-induced liver injury in D-galactosamine-sensitized mice requires secreted TNF- $a$ and the TNF-p55 receptor. Am J Physiol Regul Integr Comp Physiol 2000;278:R1202-9.
29. Hossen MJ, Kim MY, Kim JH, Cho JY. AP-1-targeted inhibition of macrophage function and lipopolysaccharide/D-galactosamineinduced hepatitis by Phyllanthus acidus methanolic extract. Am J Chin Med 2015;43:1137-58.

30. Dejager $L$, Libert $C$. Tumor necrosis factor alpha mediates the lethal hepatotoxic effects of poly $(\mathrm{l}: \mathrm{C})$ in D-galactosamine-sensitized mice. Cytokine 2008;42:55-61.

31. Kim YW, West XZ, Byzova TV. Inflammation and oxidative stress in angiogenesis and vascular disease. J Mol Med (Berl) 2013;91: 323-8.

32. Lee JP, Li YC, Chen HY, Lin RH, Huang SS, Chen HL, Kuan PC, Liao MF, Chen CJ, Kuan YH. Protective effects of luteolin against lipopolysaccharide-induced acute lung injury involves inhibition of MEK/ERK and PI3K/Akt pathways in neutrophils. Acta Pharmacol Sin 2010;31:831-8.

33. Park CM, Jin KS, Cho CW, Lee YW, Huh GH, Cha YS, Song YS. Luteolin inhibits inflammatory responses by down-regulating the JNK- NFKB and AP-1 pathways in TNF-a activated HepG2 cells. Food Sci Biotechnol 2012;21:279-83.

34. Shimoi K, Okada H, Furugori M, Goda T, Takase S, Suzuki M, Hara $\mathrm{Y}$, Yamamoto $\mathrm{H}$, Kinae $\mathrm{N}$. Intestinal absorption of luteolin and luteolin 7-O-beta-glucoside in rats and humans. FEBS Lett 1998;438: 220-4.

35. Murota K, Shimizu S, Miyamoto S, Izumi T, Obata A, Kikuchi M, Terao J. Unique uptake and transport of isoflavone aglycones by human intestinal caco-2 cells: comparison of isoflavonoids and flavonoids. J Nutr 2002;132:1956-61.

36. Hollman PC, Katan MB. Health effects and bioavailability of dietary flavonols. Free Radic Res 1999;31 Suppl:S75-80.

37. Ross JA, Kasum CM. Dietary flavonoids: bioavailability, metabolic effects, and safety. Annu Rev Nutr 2002;22:19-34. 